\title{
Design of multi-layer anti-reflection coating for terrestrial solar panel glass
}

\author{
B GEETHA PRIYADARSHINI ${ }^{1, *}$ and A K SHARMA ${ }^{2}$ \\ ${ }^{1}$ Nanotech Research Innovation \& Incubation Centre, PSG Institute of Advanced Studies, \\ Peelamedu, Coimbatore 641004, India \\ ${ }^{2}$ National Centre for Photovoltaic Research \& Education, Department of Electrical Engineering, \\ Indian Institute of Technology, Bombay 400 076, India
}

MS received 14 September 2015; accepted 10 December 2015

\begin{abstract}
To date, there is no ideal anti-reflection (AR) coating available on solar glass which can effectively transmit the incident light within the visible wavelength range. However, there is a need to develop multifunctional coating with superior anti-reflection properties and self-cleaning ability meant to be used for solar glass panels. In spite of self-cleaning ability of materials like $\mathrm{TiO}_{2}$ and $\mathrm{ZnO}$, these coatings on glass substrate have tendency to reduce light transmission due to their high refractive indices than glass. Thus, to infuse the anti-reflective property, a low refractive index, $\mathrm{SiO}_{2}$ layer needs to be used in conjunction with $\mathrm{TiO}_{2}$ and $\mathrm{ZnO}$ layers. In such case, the optimization of individual layer thickness is crucial to achieve maximum transmittance of the visible light. In the present study, we propose an omni-directional anti-reflection coating design for the visible spectral wavelength range of $400-700 \mathrm{~nm}$, where the maximum intensity of light is converted into electrical energy. Herein, we employ the quarter wavelength criteria using $\mathrm{SiO}_{2}, \mathrm{TiO}_{2}$ and $\mathrm{ZnO}$ to design the coating composed of single, double and triple layers. The thickness of individual layers was optimized for maximum light transmittance using essential Mcleod simulation software to produce destructive interference between reflected waves and constructive interference between transmitted waves.
\end{abstract}

Keywords. Anti-reflection; thin film; thickness; refractive index; interference; optical impedance.

\section{Introduction}

In photovoltaic (PV) module, the cover glass surface reflects more than $4 \%$ of incident light across the spectrum which needs to be effectively utilized for energy conversion. Additional loss due to soiling has become more common issue in substantial polluted areas due to dust coverage on solar panels [1]. Presence of microdepressions leads to the accumulation of dust and rain water droplets over the glass surface causing corrosion in solar panels. The optical parameters are affected by soiling effects which subsequently reduces the energy generation due to low light transfer thereby causing additional loss in the overall efficiency (1.17-1.42\%) [2]. Therefore, under such circumstances, regular cleaning of solar modules becomes indispensable which might lead to abrasive cleaning of glass resulting into further development of scratches on the glass panels. To overcome these issues, glass surface properties can be modified by applying a suitable protective anti-reflective (AR) coating which possesses high transparency to visible light and self-cleaning ability.

However, the biggest challenge of this approach is the availability of material with refractive index lower than that of glass $(n<1.5)$. An ideal single-layer AR coating on a

\footnotetext{
*Author for correspondence (bgp@psgias.ac.in, geethapriyadarshini@gmail.com)
}

glass surface in air ambient would require a material with a refractive index of 1.2 and there is no single material available with low refractive index. However, reflective losses can be minimized with single-layered coating of thickness equal to quarter of wavelength causing destructive interference of the reflected light waves from coated surface and substrate. Generally, magnesium fluoride $\left(\mathrm{MgF}_{2}\right)$ is used for single layer AR due to low refractive index of 1.14, however, less durable when used in outdoor environment [3]. Alternatively, porous $\mathrm{SiO}_{2}$ is preferred due to reasonably low refractive index $(n<1.45)$ with good environmental stability and durability. Recently, authors have demonstrated improvement in light trapping in $\mathrm{Si}$ solar cells with maximum reduction in reflection by $85-100 \%$ in visible region of 400-550 nm when amorphous $\mathrm{BaTiO}_{3}$ thin film with refractive index of 2.02-1.91 were deposited over Si solar cells [4]. However, single layer anti-reflection coating can minimize reflections at one specific wavelength typically at normal incidence which limits its use for broad visible range. In addition, they are inherently incapable to exhibit spectrally broadband reduction in reflectance over a wide range of angles of incidence.

Recently, multilayer AR design seems to be more promising due to the fact that material with refractive index lower than glass is no longer required [5,6]. Through proper selection of the material and layer thickness, the interference of light can be controlled by minimizing the reflection losses. 
Two or more materials of low (1.45-1.52) and high (2.0-2.3) refractive indices can be preferred depending on the level of anti-reflection required for specific application. In turn, these high refractive indices layers used as sequential layers along with low refractive index material is known to enhance the transparency of glass.

Particularly, $\mathrm{TiO}_{2}$ and $\mathrm{ZnO}$ thin films have a wide optical band gap of 3.05 and $3.3 \mathrm{eV}$, respectively, shows excellent optical transmittance in visible and near infra-red region. In spite of self-cleaning ability of $\mathrm{TiO}_{2}$ and $\mathrm{ZnO}$, these coating on glass substrate has tendency to increase the reflectivity. To improve the anti-reflective property, a low refractive index material like $\mathrm{SiO}_{2}$, which would enhance the light transmission has to be used in conjunction with $\mathrm{TiO}_{2}$ and $\mathrm{ZnO}$ layers. Ye et al [7] developed abrasion resistant, hydrophobic $\mathrm{SiO}_{2} / \mathrm{TiO}_{2} / \mathrm{SiO}_{2}-\mathrm{TiO}_{2}$ triple coating with high transmittance of $98.4 \%$ at visible region using dip-coating method. Similarly, $\mathrm{Li}$ et al [8] designed and developed highly durable non-quarter wave double-layer $\mathrm{TiO}_{2}-\mathrm{SiO}_{2}$ and $\mathrm{ZrO}_{2}-\mathrm{SiO}_{2}$ using sol-gel technique which revealed transmittance up to 94.4 and $94.31 \%$, respectively. Gao et al [9] developed highly transparent and UV-resistant superhydrophobic arrays of $\mathrm{SiO}_{2}-\mathrm{ZnO}$ nanocomposite, coated on glass substrates showing minimal impact on solar cell device performance under AM1.5G illumination.

Self-cleaning phenomenon is based on the contact angle $\theta_{\mathrm{c}}$, if $\theta_{\mathrm{c}}$ approaching to $0^{\circ}$ it is known as super hydrophilic and if $\theta_{\mathrm{c}}$ close to $150^{\circ}$ then known as super hydrophobic. Hydrophilic coating carries away the dirt and other impurities with water droplets, whereas in case of hydrophobic coating the water droplets slide and roll over the surfaces thereby cleaning them. However, to achieve self-cleaning, a film not only must be super hydrophilic but also must be able to induce photocatalytic oxidative decomposition. The hydrophilic coatings doped with suitable metal oxides, chemically breaking down the complex dirt deposits by sunlight-assisted cleaning mechanism [10].

The self-cleaning ability of $\mathrm{TiO}_{2}$ and $\mathrm{ZnO}$ coatings applied on solar glass are well studied. Zhang et al [11] reported the nanosized $\mathrm{TiO}_{2}$ particles coated on the surface of the $\mathrm{SiO}_{2}$ layer shows excellent surface wettability with $\sim 1^{\circ}$ contact angle and $99 \%$ transmission despite the high refractive index of $\mathrm{TiO}_{2}$. Medina-Valtierra et al [12] demonstrated that the doping of metal in $\mathrm{TiO}_{2}$ enhancing the self-cleaning property of nanostructured thin film. Ebert and Bhusan [13] studied self-cleaning and transparent properties of $\mathrm{SiO}_{2}, \mathrm{ZnO}$ and ITO nanoparticle coatings on glass and plastic substrates (polycarbonate and PMMA) and found superhydrophobic nature $\theta_{\mathrm{c}}\left(>150^{\circ}\right)$ and high transmittance of visible light ( $T \sim 90 \%$ ) in terms of wettability and transmittance.

Development of multi-layer coating with optimized layer thickness using analytical approach is a difficult challenge and adds complexity to the fabrication process. Therefore, great emphasis should be laid on selection of materials and design of layers is crucial aspect prior to methodical evaluation of the coating for the desired requirement. With this aim, we intend to design multifunctional coatings with superior anti-reflection properties in broadband range using cost-effective and high refractive index materials like $\mathrm{TiO}_{2}$ and $\mathrm{ZnO}$. In addition, these materials possess high selfcleaning ability to modify the surface wetabillity of the glass substrates which is another important parameter for reducing the reflection losses due to soiling. The design of the antireflection coating was made to achieve minimum reflectance and maximum transmittance in the wavelength range of 400 $700 \mathrm{~nm}$ using Essential Macleod commercial software package [14]. This work is focussed on the development of a single, double and triple layers broadband designs. Therefore, the efficiency of PV module can be improved by depositing sequential layers of metal oxides possessing antireflection properties in broadband range.

\section{Methodology}

To achieve an optimal anti-reflection effect resulting in zero net reflection in the spectral range of interest $400-700 \mathrm{~nm}$, the amplitude of the reflected beam at the air-coating interface and coating-substrate interface has to be equal and exactly one-half wave $\left(180^{\circ}\right)$ out of phase, resulting in destructive interference. This condition is met by the optical thickness of the anti-reflection coating layer $(t)$ must be close to a quarter of the wavelength $(\lambda)$ at which zero reflectance should occur (i.e., the reflected wave must be out of phase by $\pi$ ) expressed by equation (1):

$$
t=\frac{\lambda}{4 n_{1}}+m \frac{\lambda}{2 n_{1}} \quad(m=0,1,2,3 \ldots),
$$

where $n_{1}$ is the refractive index of the thin-film layer [15]. Second condition is that the magnitude of front surface reflections must be equal to the magnitude of the sum of all other partial reflections in the thin-film layer. This condition can be met when refractive index of the single layered coating $\left(n_{1}\right)$ equals to the square root of the product of the refractive indices of substrate $\left(n_{\mathrm{s}}\right)$ and medium $\left(n_{0}\right)$ expressed by equation (1) [16]:

$$
n_{1}=\sqrt{n_{\mathrm{s}} n_{0}} .
$$

However, the region of low reflectivity for a single-layer optical coating occurs at thickness of $\lambda / 4 n_{1}$ which cannot cover the entire absorption width over a $400-700 \mathrm{~nm}$ visible range. It is often difficult to choose intermediate index materials with refractive indices that are close to ideal. To obtain the equivalent optical properties of an intermediateindex layer, a stack consisting of alternating layers of lowand high-index materials are used. The optimal performance is obtained when the refractive index of each layer is the geometric mean of the refractive indices of its two neighbouring layers. Each layer adds to a minimum to the reflectance spectrum, which can be used to increase the absorption width of the low reflectivity region. The multilayer stack design requires optimization of the individual layer thicknesses based on quarter-wavelength. 
In this study, we use Essential Mcleod software which models the optical coating using the transfer matrix method to predict propagation of electromagnetic wave through the thin film stack. Optical interference matrix is an effective way to calculate reflectivity in multilayer thin film. Under the situation of zero degree incident angle of light, considering the optical thin film system of $N$ layers, $n_{\mathrm{j}}$ is the refractive index and $k_{\mathrm{j}}$ the extinction coefficient, $d_{\mathrm{j}}$ the thickness of each layer, respectively, and $n_{0}$ the refractive index of air $\left(n_{0}=1\right)$. The reflectance in multilayers is calculated by the optical admittance $(Y)$ which is the ratio of the total tangential magnetic $(C)$ and electric fields $(B)$. According to the refractive index and thickness of each layer, interference matrix of each layer can be determined using characteristic matrix formulation with the following equation (3) [17]:

$$
\left[\begin{array}{l}
B \\
C
\end{array}\right]=\left\{\prod_{r=1}^{q}\left[\begin{array}{cc}
\cos \delta_{\mathrm{r}} & \left(i \sin \delta_{\mathrm{r}}\right) / n_{\mathrm{r}} \\
i n_{\mathrm{r}}\left(\sin \delta_{\mathrm{r}}\right) & \cos \delta_{\mathrm{r}}
\end{array}\right]\right\}\left[\begin{array}{c}
1 \\
n_{\mathrm{m}}
\end{array}\right],
$$

where $\delta_{\mathrm{r}}=2 \pi N d \cos \vartheta / \lambda$ is the effective optical thickness of the layer at a given wavelength and $n_{\mathrm{m}}$ is the substrate refractive index. If $q$ is the layer next to the substrate, then the order is

$$
\left[\begin{array}{l}
B \\
C
\end{array}\right]=\left[M_{1}\right]\left[M_{2}\right] \ldots \ldots\left[M_{q}\right]\left[\begin{array}{c}
1 \\
n_{\mathrm{m}}
\end{array}\right]
$$

$M_{1}$ indicates the matrix associated with layer 1 , and so on. As in the case of a single surface, $n_{\mathrm{o}}$ must be real for reflectance and transmittance should have a valid meaning. Using equations (3) and (4), reflectance $(R)$ is derived to be:

$$
R=\left(\frac{n_{0} B-C}{n_{0} B+C}\right)\left(\frac{n_{0} B-C}{n_{0} B+C}\right)^{*} .
$$

In this study, anti-reflection coating design was optimized using $\mathrm{SiO}_{2}, \mathrm{ZnO}$ and $\mathrm{TiO}_{2}$ layers to minimize the single

\begin{tabular}{|c|c|c|c|c|c|}
\hline & Design & Materials & Refractive index & $\begin{array}{l}\text { Extinction } \\
\text { co-efficient }\end{array}$ & $\begin{array}{c}\text { Coating } \\
\text { thickness (nm) }\end{array}$ \\
\hline 1 & Medium & Air & 1.00000 & 0.00000 & - \\
\hline & Substrate & Glass & 1.52083 & 0.00000 & \\
\hline 2 & Medium & Air & 1.00000 & 0.00000 & \\
\hline & Layer 1 & $\mathrm{SiO}_{2}$ & 1.46180 & 0.00000 & 87.93 \\
\hline & Substrate & Glass & 1.52083 & 0.00000 & \\
\hline 3 & Medium & Air & 1.00000 & 0.00000 & \\
\hline & Layer 1 & $\mathrm{SiO}_{2}$ & 1.46180 & 0.00000 & 87.22 \\
\hline & Layer 2 & $\mathrm{TiO}_{2}$ & 2.34867 & 0.00000 & 54.29 \\
\hline & Substrate & Glass & 1.52083 & 0.00000 & \\
\hline 4 & Medium & Air & 1.00000 & 0.00000 & \\
\hline & Layer 1 & $\mathrm{SiO}_{2}$ & 1.46180 & 0.00000 & 87.22 \\
\hline & Layer 2 & $\mathrm{ZnO}$ & 2.03718 & 0.00000 & 62.59 \\
\hline & Substrate & Glass & 1.52083 & 0.00000 & \\
\hline 5 & Medium & Air & 1.00000 & 0.00000 & \\
\hline & Layer 1 & $\mathrm{SiO}_{2}$ & 1.46180 & 0.00000 & 87.22 \\
\hline & Layer 2 & $\mathrm{ZnO}$ & 2.03718 & 0.00000 & 125.17 \\
\hline & Layer 3 & $\mathrm{TiO}_{2}$ & 2.34867 & 0.00000 & 54.29 \\
\hline & Substrate & Glass & 1.52083 & 0.00000 & \\
\hline 6 & Medium & Air & 1.00000 & 0.00000 & \\
\hline & Layer 1 & $\mathrm{SiO}_{2}$ & 1.46180 & 0.00000 & 87.22 \\
\hline & Layer 2 & $\mathrm{ZnO}$ & 2.03718 & 0.00000 & 62.59 \\
\hline & Layer 3 & $\mathrm{TiO}_{2}$ & 2.34867 & 0.00000 & 54.29 \\
\hline & Substrate & Glass & 1.52083 & 0.00000 & \\
\hline 7 & Medium & Air & 1.00000 & 0.00000 & \\
\hline & Layer 1 & $\mathrm{SiO}_{2}$ & 1.46180 & 0.00000 & 87.22 \\
\hline & Layer 2 & $\mathrm{TiO}_{2}$ & 2.34867 & 0.00000 & 108.99 \\
\hline & Layer 3 & $\mathrm{ZnO}$ & 2.03718 & 0.00000 & 62.59 \\
\hline & Substrate & Glass & 1.52083 & 0.00000 & \\
\hline 8 & Medium & Air & 1.00000 & 0.00000 & \\
\hline & Layer 1 & $\mathrm{SiO}_{2}$ & 1.46180 & 0.00000 & 87.22 \\
\hline & Layer 2 & $\mathrm{TiO}_{2}$ & 2.34867 & 0.00000 & 54.29 \\
\hline & Layer 3 & $\mathrm{ZnO}$ & 2.03718 & 0.00000 & 62.59 \\
\hline & Substrate & Glass & 1.52083 & 0.00000 & \\
\hline
\end{tabular}
surface reflection on glass for wavelength in the range of

Table 1. Coating design. 
400-700 nm. The values of refractive indices for $\mathrm{SiO}_{2}, \mathrm{ZnO}$ and $\mathrm{TiO}_{2}$ were selected using materials library database. To obtain the optical spectra, the reference wavelength was fixed at $510 \mathrm{~nm}$ with normal angle of incidence. To understand the effect of incident angle on anti-reflection properties, the angle of incidence was varied from 0 to $90^{\circ}$, while designing the layer thickness and subsequently reflection and transmittance spectras were simulated. Table 1 shows the details of the design parameters chosen for the present investigation.

\section{Results and discussion}

The reflectance and transmittance spectras were plotted as show in figure $1 \mathrm{a}$ and $\mathrm{b}$, respectively. The uncoated glass covered with thin layer of air shows single-side reflectance of about $8 \%$ and transmittance of about $92 \%$ in wavelength range of 400-700 nm. For a single layered $\mathrm{SiO}_{2}$ coating, the thickness obtained for reducing reflection to $3.0-3.2 \%$ is $87.93 \mathrm{~nm}$ with marginal increase in transmittance to $96.8-97.1 \%$. For designs 3 and 4, the double layer
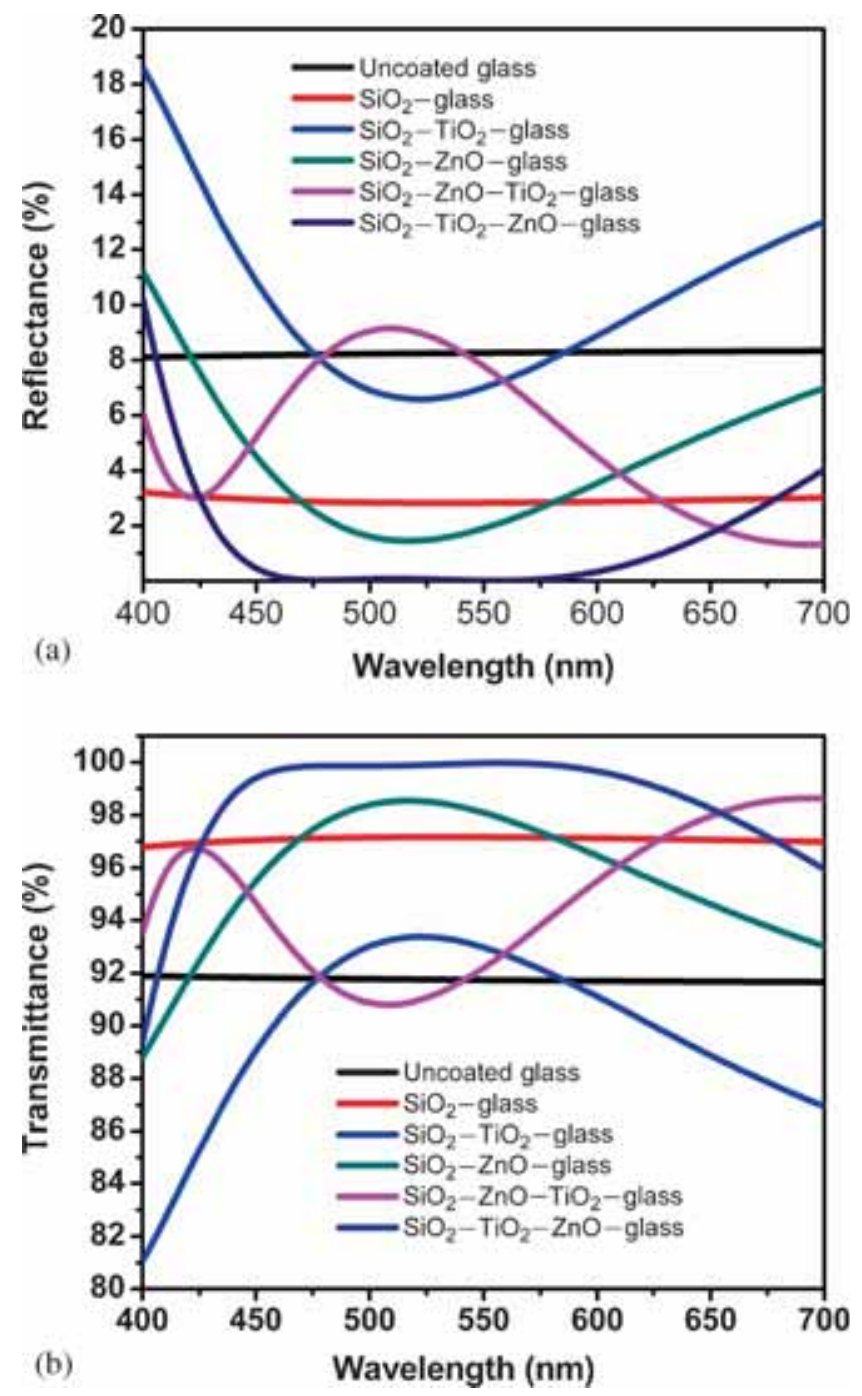

Figure 1. The modelled performance of single, double and triple layer coatings. coating thickness obtained was based on quarter-quarter (Q-Q) wavelength theory. The surface reflection increased to $13.0-19.0 \%$, while transmittance was decreased to $81-$ $93.37 \%$ for simulated double layer coating comprising of $87.22 \mathrm{~nm} \mathrm{SiO} 2$ and $54.29 \mathrm{~nm} \mathrm{TiO}_{2}$ on glass substrate. Interestingly, double layer coating composed of $87.22 \mathrm{~nm} \mathrm{SiO} 2$ and $62.59 \mathrm{~nm} \mathrm{ZnO}$ on glass, yielded a relatively lower reflectance of 7-11.5\% and high transmittance of 89-98.45\% in entire 400-700 $\mathrm{nm}$ wavelength range. This is explained by the Schuster diagram shown in figure 2 with $\mathrm{SiO}_{2}$ as the low index of 1.46, while an upper bound range of refractive indices for $\mathrm{TiO}_{2}$ and $\mathrm{ZnO}$ of 2.34 and 2.03, respectively. The area of shaded region shows the possible values of film indices for a quarter-quarter coating composed of $\mathrm{SiO}_{2}-\mathrm{TiO}_{2}$ and $\mathrm{SiO}_{2}-\mathrm{ZnO}$ on glass. The narrow range of indices in case of $\mathrm{SiO}_{2}-\mathrm{ZnO}$, contributes to the decrease in the reflectivity when compared to $\mathrm{SiO}_{2}-\mathrm{TiO}_{2}$ coating.

However, in both these cases typical ' $\mathrm{V}$ ' shaped characteristic curve was observed signifying that the anti-reflection property can be realized only at narrow wavelength range. The reflectance value was lowest for $\mathrm{SiO}_{2}-\mathrm{ZnO}$ double layer when compared to single $\mathrm{SiO}_{2}$ layer and $\mathrm{SiO}_{2}-\mathrm{TiO}_{2}$ double layer. In case of triple layer coating comprised of $87.22 \mathrm{~nm}$ $\mathrm{SiO}_{2}-62.59 \mathrm{~nm} \mathrm{ZnO}-54.29 \mathrm{~nm} \mathrm{TiO}_{2}$ (design 6) on glass, the reflectance increases to $9 \%$ at $510 \mathrm{~nm}$ which is more than the uncoated glass. However, triple coating composed of $87.22 \mathrm{~nm} \mathrm{SiO}_{2}-54.29 \mathrm{~nm} \mathrm{TiO}_{2}-62.59 \mathrm{~nm} \mathrm{ZnO}$ coating (design 8) on glass showed a remarkable decrease in reflectance of $<1 \%$ with high transmittance of $99 \%$ in the broad range of wavelength from 450 to $700 \mathrm{~nm}$. This suggests that triple coating would be ideal for improving antireflection properties of PV glass modules in the broad range of visible portion of incident light.

Single layer $\mathrm{SiO}_{2}$ coating on glass benefited with lowering the reflectance to $3 \%$ and transmittance to $\sim 97 \%$ in

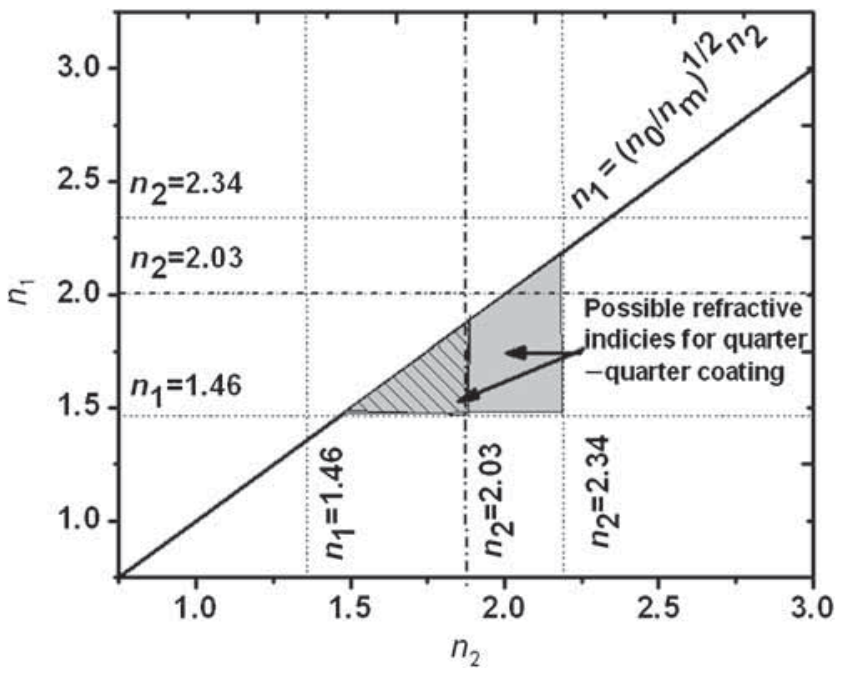

Figure 2. A Schuster diagram showing possible refractive indices for quarter-quarter wavelength coating composed of $\mathrm{SiO}_{2}\left(n_{1}=\right.$ $1.46)-\mathrm{ZnO}\left(n_{2}=2.03\right)$ and $\mathrm{SiO}_{2}\left(n_{1}=1.46\right)-\mathrm{ZnO}\left(n_{2}=2.34\right)$ on glass. 
wavelength of 400-700 nm. Inclusion of $\mathrm{TiO}_{2}$ with $\mathrm{SiO}_{2}$ in double layer has resulted minima at wavelength $520 \mathrm{~nm}$ but resulted in higher reflectance towards lower and higher wavelengths, while inclusion of $\mathrm{ZnO}$ with $\mathrm{SiO}_{2}$ resulted in decrease in reflectance and increment in transmittance in relatively broad wavelength range of $470-570 \mathrm{~nm}$. Triple layer $\mathrm{SiO}_{2}(1.46) / \mathrm{ZnO}(2.03) / \mathrm{TiO}_{2}(2.34)$ stack with $n_{\text {air }}<n_{\mathrm{SiO}_{2}}<$ $n_{\mathrm{ZnO}}<n_{\mathrm{TiO}_{2}}>n_{\text {glass }}$ showed two minima in reflection (first at $420 \mathrm{~nm}$ and second at $700 \mathrm{~nm}$ ) and one maxima at $520 \mathrm{~nm}$ which does not meet the requirements of minimum reflection in 450-650 nm wavelength corresponding to maximum photon flux in the solar spectrum on earth. It was observed that when second and third layer was interchanged in the stack i.e., $n_{\text {air }}<n_{\mathrm{SiO}_{2}}<n_{\mathrm{TiO}_{2}}>n_{\mathrm{ZnO}}>n_{\text {glass }}$ minimum reflection with transmission $>90 \%$ in $450-650 \mathrm{~nm}$ wavelength range. From these findings it is observed that by having a high refractive index layers sandwiched between two low index layer $\left(n_{\mathrm{SiO}_{2}}<n_{\mathrm{TiO}_{2}}>n_{\mathrm{ZnO}}\right)$ leads to minimum reflections and maximum transmittance when compared to the graded index layers $\left(n_{\mathrm{SiO}_{2}}<n_{\mathrm{ZnO}}<n_{\mathrm{TiO}_{2}}\right)$.

According to Thetford vector model [18], the two amplitude reflection coefficients are computed using multi-layer stack split into two parts on either side of the middle layer. At the reference wavelength of $\lambda_{0}=550 \mathrm{~nm}$, the quarter-wave length thickness of the multi-layer coating made the reflectance equal. A second value of wavelength $\left(\lambda_{1}\right)$ shorter
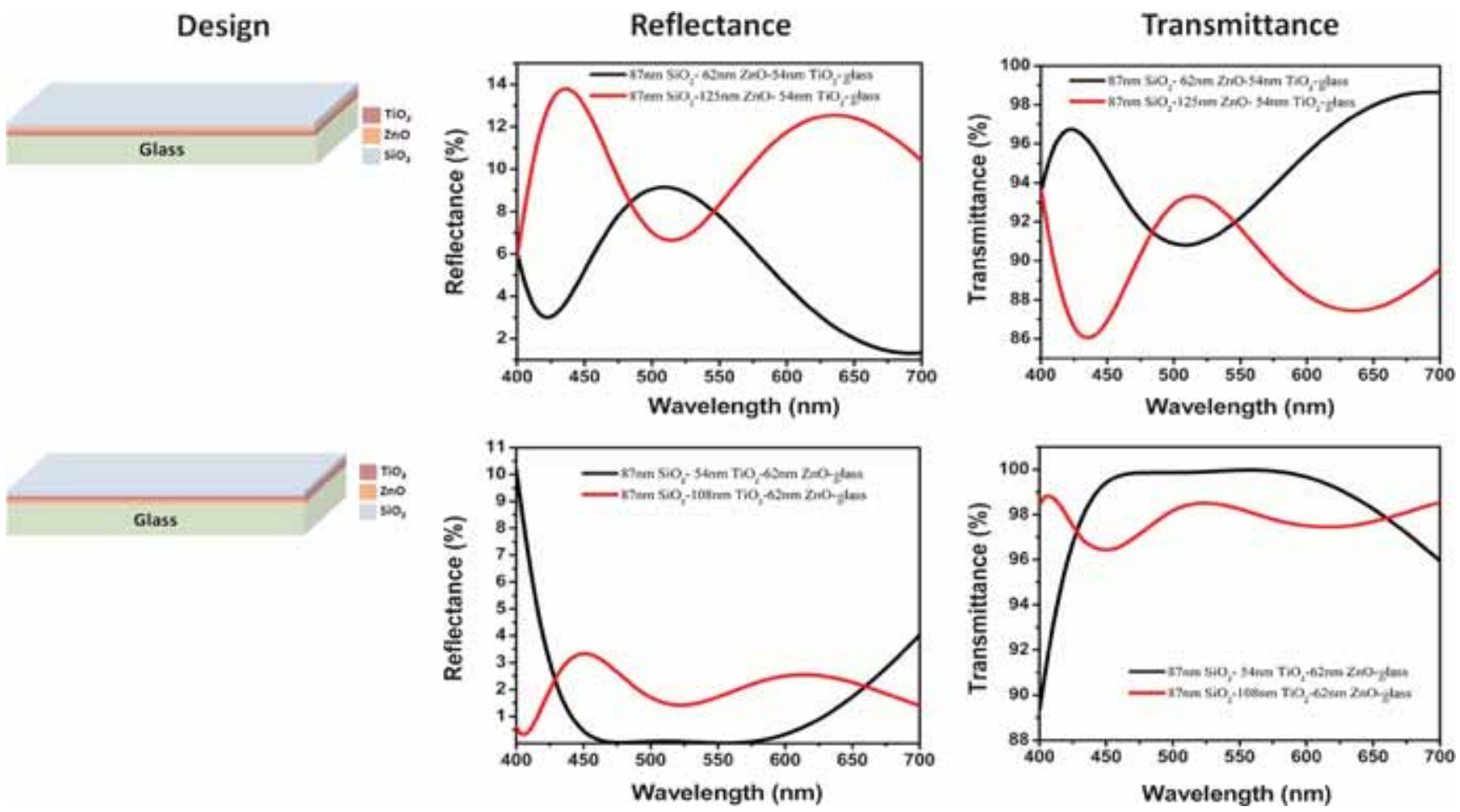

Figure 3. Computed spectra of reflectance and transmittance of a glass with $\mathrm{SiO}_{2}-\mathrm{ZnO}-\mathrm{TiO}_{2}$ and $\mathrm{SiO}_{2}-\mathrm{TiO}_{2}-\mathrm{ZnO}$ coatings on the top with thickness equivalent to Q-Q-Q and Q-H-Q wavelengths.

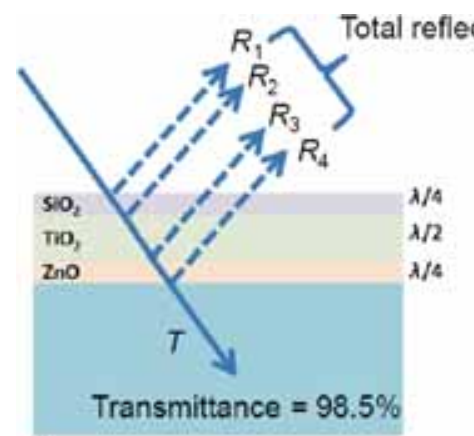

(a)

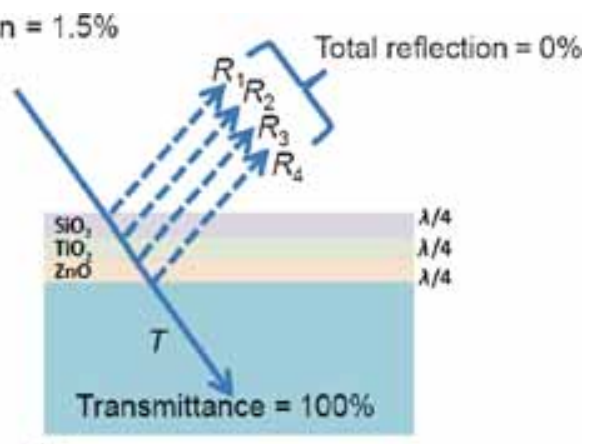

(b)

Figure 4. Schematic representation of reflection and transmittance occurring in (a) QHQ and (b) QQQ designs composed of $\mathrm{SiO}_{2}$ (top layer) $-\mathrm{TiO}_{2}$ (middle layer) - $\mathrm{ZnO}$ (bottom layer) triple coatings. 
$\mathrm{SiO}_{2}$ glass: rellectance (mean-pol)

SiO2- glass: transmittance (mean-pol)
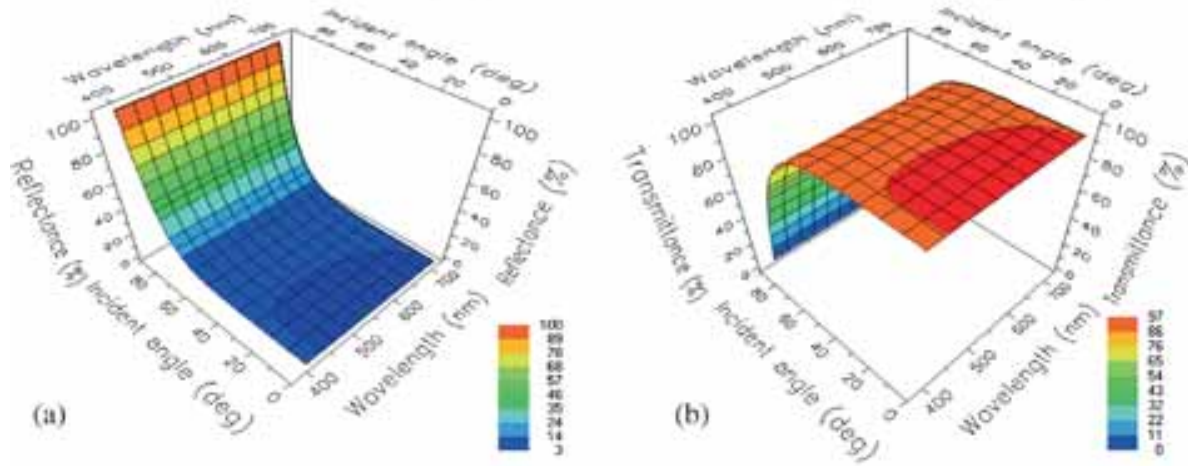

$\mathrm{SiO}_{2}-\mathrm{ZnO}$-glass: reflectance (mean-pol)

$\mathrm{SiO}_{2}-\mathrm{ZnO}$-glass: transmittance (mean-pol)
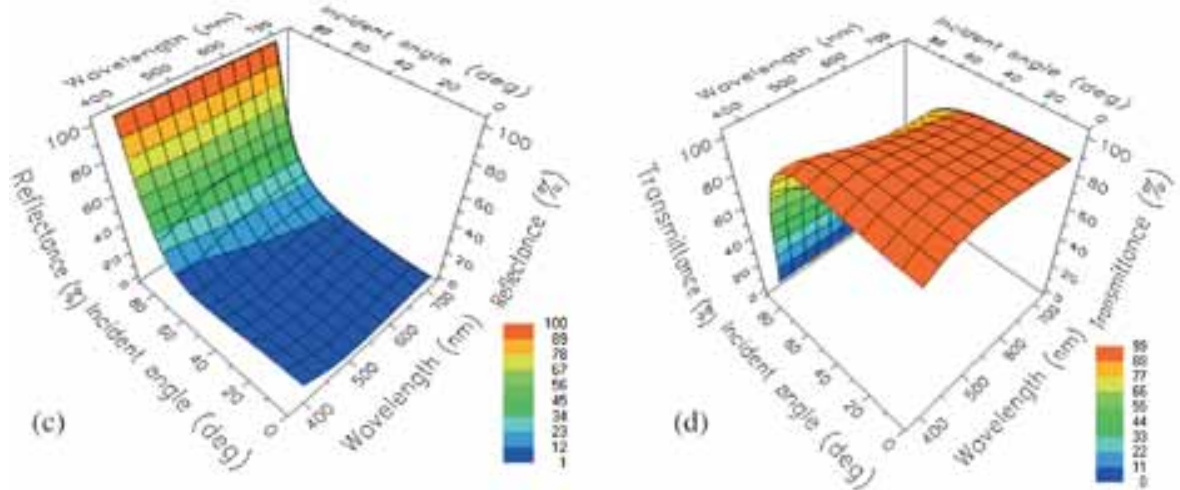

$\mathrm{SiO}_{2}-\mathrm{TiO}_{2}-\mathrm{ZnO}$ (QHQ): reflectance (mean-pol)

$\mathrm{SiO}_{2}-\mathrm{TiO}_{2}-\mathrm{ZnO}(\mathrm{QHQ}$ ): transmittance (mean-pol)
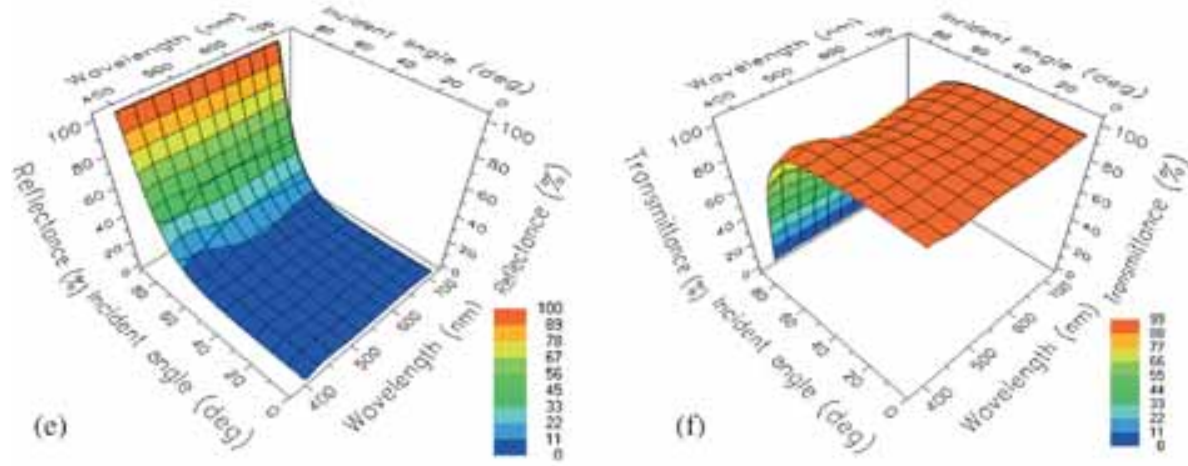

$\mathrm{SiO}_{2}-\mathrm{TiO}_{2}-\mathrm{ZnO}(\mathrm{QQQ})$ ) reflectance (mean-pol)

$\mathrm{SiO}_{2}-\mathrm{TiO}_{2}-\mathrm{ZnO}(\mathrm{QQQ})$ : transmittance (mean-pol)
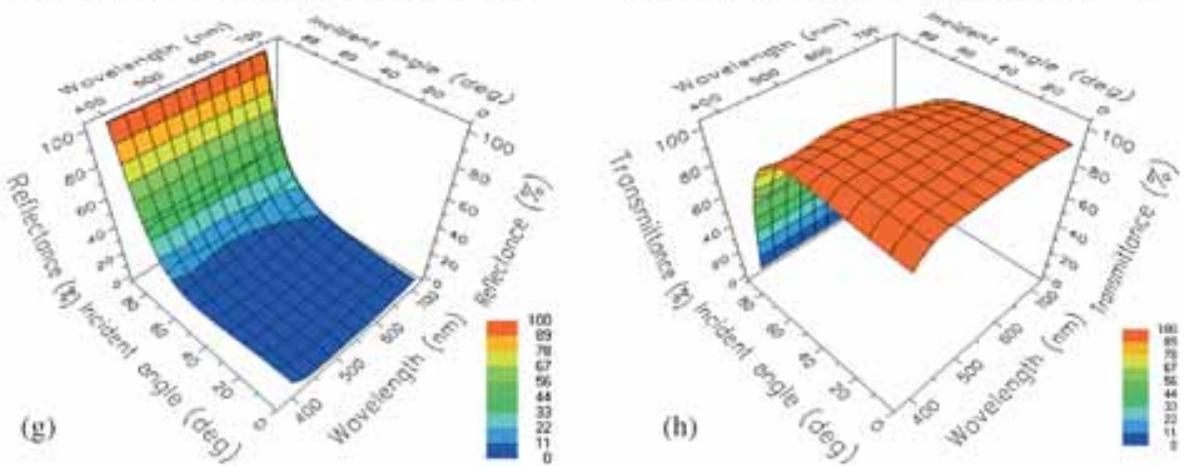

Figure 5. Computed 3D spectra of reflectance and transmittance (a-b) $\mathrm{SiO}_{2}$-coated glass, (c-d) $\mathrm{SiO}_{2}-\mathrm{ZnO}$ coated glass, (e-f) $\mathrm{SiO}_{2}-\mathrm{TiO}_{2}-\mathrm{ZnO}$ coating with thickness equivalent to $\mathrm{Q}-\mathrm{H}-\mathrm{Q}$ wavelength and $(\mathbf{g}-\mathbf{h}) \mathrm{SiO}_{2}-\mathrm{TiO}_{2}-\mathrm{ZnO}$ coating with thickness equivalent to Q-Q-Q wavelength. 
than the first, at which the reflectance is equal, obtained with the expression related to change in reflectance with wavelength for each of the two structures. Therefore, phase condition was satisfied to give zero reflectance for the complete coating at the wavelength range of $450-650 \mathrm{~nm}$. It is worth mentioning here that the thickness of the middle layer is a key factor to obtain the zero reflectance by satisfying the phase conditions at first and subsequent wavelengths.

Thickness of multilayer coating has a crucial role to play in minimizing the reflection from the glass surface. Herein, the thickness of the layers was chosen such that it obeys quarterhalf-quarter (Q-H-Q) and quarter-quarter-quarter (Q-Q-Q) wavelength rule to obtain resultant zero reflectance. Instead, $\mathrm{SiO}_{2}-\mathrm{ZnO}-\mathrm{TiO}_{2}$ coating on glass with thickness of each layer equivalent to Q-H-Q at $510 \mathrm{~nm}$ wavelength revealed high reflectance values of 6-14\%, while transmittance value of $86-94 \%$ (figure 3). However, relative decrease in reflectance to $0.5-9 \%$ with transmittance of $91-98 \%$ was observed for $\mathrm{Q}-\mathrm{Q}-\mathrm{Q}$ coating $\left(\mathrm{SiO}_{2}-\mathrm{ZnO}-\mathrm{TiO}_{2}\right)$.

On the other hand, $\mathrm{SiO}_{2}-\mathrm{TiO}_{2}-\mathrm{ZnO}$ coatings showed drastic improvement in the reflectance and transmittance values for both Q-H-Q and Q-Q-Q configuration in broad range of wavelength. This is due to the fact that second layer $\left(\mathrm{TiO}_{2}\right)$ has a high index of refraction than the first layer $\left(\mathrm{SiO}_{2}\right)$ and third layer $(\mathrm{ZnO})$ and besides the optical thickness of $\mathrm{TiO}_{2}$ is about quarter of the reference wavelength which lowers the reflectance down to $0.5-2.0 \%$. In present case, the relative differences in refractive indices of middle and bottom layers have led to greater optical impedance mismatch at the layer interfaces with different angle of internal reflections and thereby broadening the reflectance band. Further, the thickness of intermediatev layer of quarter-wavelength increases the optical impedance mismatch leading to higher minimum reflectance as in the case of $\mathrm{Q}-\mathrm{Q}-\mathrm{Q}$ coating design. A schematic shown in figure 4 represents the comparison of optical events taking place in $\mathrm{SiO}_{2}-\mathrm{TiO}_{2}-\mathrm{ZnO}$ triple layer based on Q-Q-Q and Q-H-Q designs.

Given that the triple layer coating show very good suppression of reflectance, further we computed the mean polarized reflectance and transmittance spectra at incidence angle varying from 0 to $90^{\circ}$ to understand its dependence on reflectance and transmittance spectra for simulated layers (figure 5). A comparison of different coatings shows that $\mathrm{SiO}_{2}-\mathrm{TiO}_{2}-\mathrm{ZnO}$ coating clearly outperforms the single- and double-layered coatings in reducing the reflection of meanpolarized incident light, especially in the visible wavelength 400-700 nm regime. Similarly, in the case of transmittance spectra, computed for different layer thicknesses of $\mathrm{SiO}_{2}-$ $\mathrm{TiO}_{2}-\mathrm{ZnO}$ coating, Q-Q-Q wavelength coating outperforms when compared to $\mathrm{Q}-\mathrm{Q}$ double and $\mathrm{Q}-\mathrm{H}-\mathrm{Q}$ triple coatings at wide range of incident angles. To validate these simulation results, experiments are being carried out by carefully controlling the individual layer thickness using magnetron co-sputtering technique. Optimization of process parameters for desired layer thickness will be carried out using response surface methodology.

\section{Conclusions}

The most interesting feature of the proposed design is the relatively small physical thickness of $\sim 206 \mathrm{~nm}$, making them feasible for practical deposition experiments. A particular feature of this design is that the refractive indices are confined between the high and low index components, which rules out the requirement for a material with refractive index close to that of the ambient (air). From this study, it is concluded that the thickness and refractive index of intermediate layer in $\mathrm{Q}-\mathrm{Q}-\mathrm{Q}$ or $\mathrm{Q}-\mathrm{H}-\mathrm{Q}$ play a dominant role in effectively cutting down the reflections through destructive interference of the reflected light. In addition, Q-Q-Q design is more appropriate for minimizing front surface reflections of glass. The layers proposed herein are standard materials for an optical coating and can be fabricated by simple deposition techniques. The designed coatings possess an ability to enhance the optical characteristics of solar photovoltaic module surface in broadband range. Therefore, such hybrid systems are highly interesting candidates and practically affordable for omni-directional anti-reflection coatings suitable for terrestrial solar panel.

\section{References}

[1] Faustini M, Nicole L, Boissiere C, Innocenzi P, Sanchez C and Hrosso D 2010 Chem. Mater. 224406

[2] Mejia F A and Kleissl J 2013 Sol. Energy 95357

[3] Kaminski P M, Lisco F and Walls J M 2014 IEEE J. Photovolt. 4452

[4] Sharma A K, Geetha Priyadarshini B, Mehta B R and Kumar D 2015 RSC Adv. 559881

[5] Cox J T, Hass G and Thelen A 1962 J. Opt. Soc. Am. 52965

[6] Rancourt J D 1996 Optical thin films: user handbook (USA: McGraw-Hill Publications)

[7] Ye L, Zhang Y, Zhang X, Hu T, Ji R, Ding B and Jiang B 2013 Sol. Energ. Mat. Sol. C 111160

[8] Li J, Lu Y, Lan P, Zhang X, Xu W and Tan R 2013 Sol. Energy 89134

[9] Gao Y, Gereige I, El Labban A, Cha D, Isimjan T T and Beaujuge P M 2014 ACS Appl. Mater. Interfaces 62219

[10] Ganesh V A, Raut H K, Nair A S and Ramakrishna S 2011 J. Mater. Chem. 2116304

[11] Zhang X T, Sato O, Taguchi M, Einaga Y, Murakami T and Fujishima A 2005 Chem. Mater. 17696

[12] Medina-Valtierra J, Frausto-Reyes C, Ramirez-Ortiz J and Camarillo-Martínez G 2008 Ind. Eng. Chem. Res. 48598

[13] Ebert D and Bhushan B 2012 Langmuir 2811391

[14] Macleod A and Clark C 2012 Optical coating design with the essential macleod (Tucson: Thin Film Center Inc.)

[15] Hadley L N and Dennison D M 1947 J. Opt. Soc. Am. 37451

[16] Cox J T and Hass G 1968 Physics of thin films (eds) Hass G and Thun R E (New York: Academic Press)

[17] Macleod H A 2001 Thin film optical filters (London: Institute of Physics)

[18] Thetford A 1969 J. Mod. Opt. 1637 